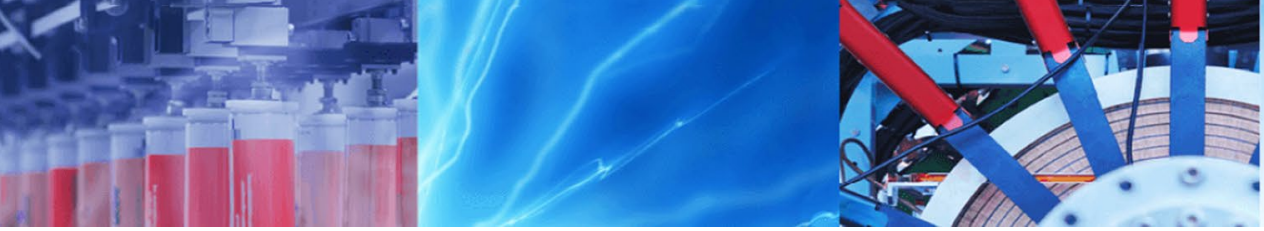

Research Article

\title{
A new approach of utility function based on fractional Gini aggregation operator for intelligent access web selection
}

\author{
Rabha W. Ibrahim ${ }^{1}$ \\ (c) Springer Nature Switzerland AG 2019
}

\begin{abstract}
The intelligent network access selection applied by the wireless communication device is designed by a decision making problem. The technique comprises getting data related to a mobile device; the data include position data connected with the mobile device. In this paper, we propose a new single-vision and multi-vision utility processes based on the utility theory. Moreover, we apply a new approach to define a new class of utility functions by utilizing the concept of Gini aggregation operator together with the idea of ordered weighted averaging. Mathematical schemes and numerical analysis slow down the ability and the practicality of our suggested simulations.
\end{abstract}

Keywords Utility function · Wireless heterogeneous · Measurement · Fractional calculus · Fractal · Fractional operator

Mathematics Subject Classification 00A71

\section{Introduction}

Normally, the intelligent network access selection (INAS) is mainly modeled by the Established Signal Strength from altered existing connecting admission nodes. However, in heterogeneous networks with corporate admission services, the INAS system goes into more complexes since numerous admission technologies evidently carry varieties of Quality of Service (QoS) and altered offering assemblies. Therefore, the INAS dresses a multi-vision decision making problem. The decision covers a chain of puzzling imitations and even trade-offs between clashing visions. The complexity of INAS is accepted as a nonlinear optimization issue [1]. Explicitly, it can be written off as an NP-hard optimization problem (non-deterministic polynomial-time hardness). This type of problems can be observed in polynomial time. A common optimal result does not appear in the web diversity context because each consumer has his own favorites. Selecting an accessible web that satisfies all majors may possibly indicate challenging as some visions might crash. One can select the discounted access web while another can communicate with the highest performing one. Consequently, consumer favorites translate the complexity of the essential multiple-vision technique. The consumer favorites imply the deep relationship between the proven vision (criteria, attribute or object) and the web selection attitude. Precisely, the favorite need is a practiced weight that consumers deal with each vision subject to their provisions. Once the vision accepted and the favorites fixed, we demand a method to compare between these accesses webs to categorize the most proper one. The obligation of this performance is the central focus of this work.

The current solution of web selection is to employ a recording method based on the theory of utility function. The rudiments of this theory were offered and established by Neumann and Morgenstern [2]. Since then, many other developments and conversation of the theory have been appreciated. In microeconomics, the device of utility idea indicates the ability of facilities or goods to achieve a human necessity. Mathematically, it is associated to a term, which is entitled the utility function (UF). Different

Rabha W. Ibrahim, rabhaibrahim@yahoo.com | ${ }^{1}$ Cloud Computing Center, University Malaya, Kuala Lumpur, Malaysia.

SN Applied Sciences (2019) 1:1126| https://doi.org/10.1007/s42452-019-1003-5 
Table 1 Structures of different types of Utility functions

\begin{tabular}{|c|c|c|}
\hline Utility design & Formula & References \\
\hline Linear & $\begin{cases}0 & \text { if } \chi<\chi_{\min } \\
\frac{\chi-\chi_{\min }}{\chi_{\max }-\chi_{\min }} & \text { if } \chi_{\min } \chi<\chi_{\max } \\
1 & \text { otherwise }\end{cases}$ & [7] \\
\hline Logarithm & $\ln (\chi) ;$ or $\ln (1+c \chi)$ & {$[5]$} \\
\hline Entropy & $\begin{array}{l}\frac{1}{\delta-1}\left(1-\sum_{i} \rho\left(\chi_{i}\right)\right) \\
\text { where } \rho\left(\chi_{i}\right) \text { is the probability of } \chi\end{array}$ & [8-10] \\
\hline Exponential & $\begin{array}{l}e^{\chi-\kappa},(0 \leq \chi \leq \kappa) \\
1-e^{-c \chi},(c \geq 0)\end{array}$ & $\begin{array}{l}{[6]} \\
{[11]}\end{array}$ \\
\hline Power utility & $\frac{\left(1+\frac{\chi}{b}\right)^{1-\ell}-1}{1-\ell}$ & [12] \\
\hline \multirow[t]{2}{*}{ Sigmoid } & $\frac{\left(\chi / \chi_{m}\right)^{c}}{1+\left(\chi / \chi_{m}\right)^{c}}, c>0$ & \\
\hline & $\frac{1}{1+e^{c \chi}}, c>0$ & [13] \\
\hline
\end{tabular}

consumers with various operator choices will have different utility values for a corresponding creation. Therefore, the theory of utility communicates to both single and multi- vision, where this utility can help in these positions by causing a decision scheme over the progression of adept practiced. In view of the data science, the utility $\mathbb{U}(\alpha)$ is a function from the data set of identifying creation principles $\alpha$ (by the agent) and the manager experiences into the set of real numbers. Generally, the score (total) of accesses $l$ is given by the evaluation of utility function by the formula

$\mathbb{U}_{l}=\sum_{J} \omega_{J} \mu_{J}\left(\alpha_{l \jmath}\right)$,

where $\alpha_{l j}$ indicates the price of pattern $J$ in access $l, \omega_{J}$ represents to the weight of pattern $J$ satisfying $\sum \omega_{J}=1$ and $\mu_{j}$ is normalized function $[3,4]$. The rapidity of the normalized function is to measure various aspects of transforming mechanisms to an analogous numerical design. There arise various forms of normalized function formulas to evaluate the ability of each value. For instance, the logarithmic form was assumed in [5], the exponential formula was proposed in [6] and a linear-piecewise was reflected in [7]. Recently, the utility functions are settled by utilizing Tsallis entropy [8-10]. Table 1 indicates some types of UF, linear and nonlinear formulations [11-13].

The usage of utility functions is to explain human behavior, mainly in different forms, or where there is a probability that some formal will arise. In general, how person principles consumption in one formal associated with another will be determined by the probability that the formal in the request will essentially arise. Our aim is to introduce a new mathematical model of the utility function using mixed operators Gini aggregation operator and OWA operator [14, 15]. These types of operators indicate many applications in intelligent systems such as fuzzy schemes, artificial neural networks, arrangement recognition, and data science for retrieval, extraction, discovery, recognition, expert systems, and multi-objective decision. With the increasing aggregate of electronically accessible data in data records, databases, data flows, and the necessity for an effective exploitation of such data, aggregation operators have achieved better concern [16-20].

The respite of the paper is systematized as follows. The methodology is presented in Sect. 2 . The suggested utility design, single-vision and multi-vision UF are introduced in Sect. 3 followed by discussion in Sect. 4. Conclusions are located in the last section.

\section{Methodology}

Our methodology is based on the aggregate operators and their combinations. We have the following definitions of two operators

Definition 2.1 The OWA operator $\Theta: \mathbb{R}^{k} \rightarrow \mathbb{R}$ is a mapping that has a connected weighting vector $\omega=\left(\omega_{1}, \ldots, \omega_{k}\right)^{T}$ of dimension $k$ agreeing to the following procedure

$\Theta\left(\alpha_{1}, \ldots, \alpha_{k}\right)=\sum_{j=1}^{k} \omega_{J} \beta_{J^{\prime}} \quad \sum_{j=1}^{k} \omega_{J}=1$,

where $\beta_{j}$ is the largest element of the set of the collected items $\left\{\alpha_{1}, \ldots, \alpha_{k}\right\}$.

Obviously, $\Theta$ can take its argument values of the real line, a significant distinct situation arises when the arguments are strained from the unit interval $J:=[0,1]$. For suitability, aggregation operators deliberated in this work will focus on this special situation. In addition $\Theta$ is commutative, monotonic, bounded and idempotent. The operator $\Theta$ has a generalization in term of fractional power as follows [21]

$\Theta^{\wp}\left(\alpha_{1}, \ldots, \alpha_{k}\right)=\left(\sum_{j=1}^{k} \omega_{J} \beta_{J}^{\wp}\right)^{1 / \wp}, \quad \sum_{j=1}^{k} \omega_{J}=1, \varnothing \neq 0$.

\section{Remark 2.1}

- $\varnothing=1, \Theta^{\varnothing}=\Theta$;

- $\varnothing \rightarrow \infty$ this implies $\Theta^{\wp}=\max \Theta$;

- $\varnothing \rightarrow-\infty$ this implies $\Theta^{\wp}=\min \Theta$; 
Definition 2.2 The Gini operator (mean) ${ }^{p} \Pi^{q}: J^{k} \rightarrow J$ is a mapping that has a connected weighting vector $\omega=\left(\omega_{1}, \ldots, \omega_{k}\right)^{T}$ of dimension $k$ agreeing to the following procedure

${ }^{p} \Pi^{q}\left(\alpha_{1}, \ldots, \alpha_{k}\right)=\left(\frac{\sum_{J=1}^{k} \omega_{J} \alpha_{J}^{p}}{\sum_{J=1}^{k} \omega_{J} \alpha_{J}^{q}}\right)^{\frac{1}{p-q}}, \quad \sum_{J=1}^{k} \omega_{J}=1, p>q$

where $\left\{\alpha_{1}, \ldots, \alpha_{k}\right\}$ is a collection of items.

Our contribution is that combining Definitions 2.1 and 2.2 to obtain the following generalization

Definition 2.3 The Gini-OWA operator ${ }^{p} \Delta^{q}: J^{k} \rightarrow J$ is a mapping that has a connected weighting vector $\omega=\left(\omega_{1}, \ldots, \omega_{k}\right)^{T}$ of dimension $k$ agreeing to the following procedure

${ }^{p} \Delta^{q}\left(\alpha_{1}, \ldots, \alpha_{k}\right)=\left(\frac{\sum_{J=1}^{k} \omega_{J} \beta_{J}^{p}}{\sum_{J=1}^{k} \omega_{J} \beta_{J}^{q}}\right)^{\frac{1}{p-q}}, \quad \sum_{j=1}^{k} \omega_{J}=1, p>q$

where $\beta_{J}$ is the $J$-th largest of $\left\{\alpha_{1}, \ldots, \alpha_{k}\right\}$.

By letting $\varpi_{J}:=\frac{\omega_{J} \beta_{J}^{q}}{\sum_{\jmath=1}^{k} \omega_{J} \beta_{J}^{p}}$ in Definition 2.3, we have the combination

${ }^{p} \Delta^{q}\left(\alpha_{1}, \ldots, \alpha_{k}\right)=\left(\sum_{J=1}^{k} \varpi_{J} \beta_{J}^{p-q}\right)^{\frac{1}{p-q}}, \quad \sum_{j=1}^{k} \varpi_{J}=1, p>q$.

It is clear that when $\varnothing:=p-q$, we have ${ }^{p} \Delta^{q}\left(\alpha_{1}, \ldots, \alpha_{k}\right)=\Theta^{\varnothing}\left(\alpha_{1}, \ldots, \alpha_{k}\right)$. Moreover, the operator ${ }^{p} \Delta^{q}\left(\alpha_{1}, \ldots, \alpha_{k}\right)$ achieves all properties of $\Theta$. Now in view of fractal theory [22], the fractional difference terms are for $\varepsilon>0$ (small value), and $\varnothing>0$,

$\Delta^{\wp} \Phi \approx \Gamma(1+\wp)(\Phi+\varepsilon)^{\wp}$,

we introduce the operator ${ }^{p} \Delta^{q}\left(\alpha_{1}, \ldots, \alpha_{k}\right)$ in fractal form

$$
\begin{aligned}
& \Delta^{\wp}\left(\alpha_{1}, \ldots, \alpha_{k}\right)=\sum_{J=1}^{k} \Gamma(1+\wp) \varpi_{J}\left(\alpha_{J}+\varepsilon_{J}\right)^{\wp}, \\
& \quad\left(\beta_{J}=\alpha_{J}+\varepsilon_{J}, \sum_{J=1}^{k} \varpi_{J}=1,0<p-q=\varnothing \leq 1, .\right.
\end{aligned}
$$

We call $\Delta^{\wp}\left(\alpha_{1}, \ldots, \alpha_{k}\right)$ the fractional Gini-OWA operator (FGO). Moreover, the fuzzy formula of $\bar{\Delta}^{\wp}\left(\alpha_{1}, \ldots, \alpha_{k}\right): J^{k} \rightarrow J$ can be suggested for $\bar{\alpha}_{J}:=\alpha_{J}+\varepsilon_{J}$ by
Table 2 Data to evaluate $\Delta^{\wp}$

\begin{tabular}{llll}
\hline$\alpha$ & 0.5 & 0.6 & 0.7 \\
$\omega$ & 0.5 & 0.2 & 0.3 \\
$\beta$ & 0.55 & 0.66 & 0.77 \\
\hline
\end{tabular}

$\bar{\Delta}^{\wp}\left(\alpha_{1}, \ldots, \alpha_{k}\right)=\sum_{j=1}^{k} \Gamma(1+\wp) \varpi_{J}\left(\frac{\bar{\alpha}_{J}-\bar{\alpha}_{\text {min }}}{\bar{\alpha}_{\max }-\bar{\alpha}_{\text {min }}}\right)^{\wp}$.

However, we shall consider the above formula to define our utility function (Table 2).

Example 2.1 Consider the following data: $p=1.5, q=1, k=3$, then $\varnothing=0.5$, with $\Gamma(1.5)=0.88$ and

A computation leads to

$$
\begin{aligned}
\varpi_{1} & =\frac{\omega_{1} \beta_{1}^{q}}{\sum_{J=1}^{3} \omega_{J} \beta_{J}^{p}} \\
& =\frac{0.5 * 0.55}{0.5 * 0.55^{1.5}+0.2 * 0.66^{1.5}+0.3 * 0.77^{1.5}}=0.5 ; \\
\varpi_{2} & =\frac{\omega_{2} \beta_{2}^{q}}{\sum_{J=1}^{3} \omega_{J} \beta_{J}^{p}} \\
& =\frac{0.2 * 0.66}{0.5 * 0.55^{1.5}+0.2 * 0.66^{1.5}+0.3 * 0.77^{1.5}}=0.2 ; \\
\varpi_{3} & =\frac{\omega_{3} \beta_{3}^{q}}{\sum_{J=1}^{3} \omega_{J} \beta_{J}^{p}} \quad 0.3 * 0.77 \\
& =\frac{0.5 * 0.55^{1.5}+0.2 * 0.66^{1.5}+0.3 * 0.77^{1.5}}{0.5}=0.3 .
\end{aligned}
$$

This implies

$\varpi_{1} \Gamma(1.5) \approx 0.5, \quad \varpi_{2} \Gamma(1.5) \approx 0.2, \quad \varpi_{3} \Gamma(1.5) \approx 0.3$.

Hence, we obtain

$\bar{\Delta}^{\wp}\left(\alpha_{1}, \alpha_{2}, \alpha_{3}\right)=\sum_{j=1}^{3} \Gamma(1.5) \varpi_{J}\left(\alpha_{J}+\varepsilon_{J}\right)^{\wp} \approx 1$.

Comparing with Gini-Owa operator, we get ${ }^{1.5} \Delta^{1}\left(\alpha_{1}, \alpha_{2}, \alpha_{3}\right)=0.712$, which means that the FGO is good for optimal maximization. Table 3 shows a comparison with other utility functions.

In web selection, the optimal maximization problem is the problem user's face: "how should I select my net to maximize my utility?" It is a kind of an optimal decision problem. It involves the selecting how much of each 
Table 3 Comparison with other utility functions

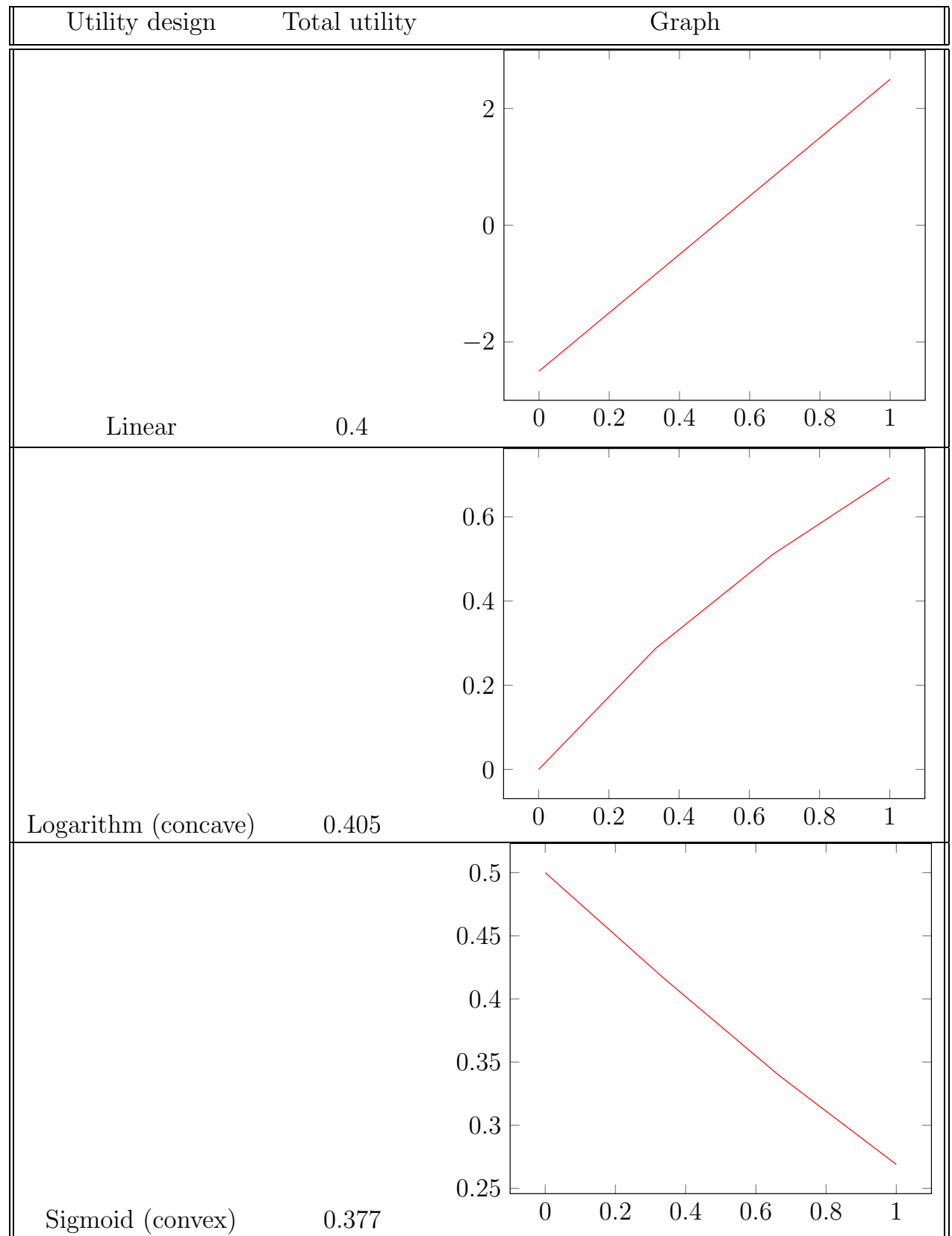

accessible service to consider, taking into account a control on total spending as well as the prices of the service.

\section{Utility function (UF)}

The theory of utility relates to both single-pattern (characteristic, criteria) and multi-pattern magnitudes. A UF is realized mathematically as a function $\mathbb{U}(\alpha)$ from a set of detecting product vision $\alpha$ (by the customer) and the customer favorites $w$ into the set of a real number. As the customer favorites connected with a set of considered pattern do not adjustment while seeing different choices, after this we indicate $\mathbb{U}(\alpha)$ as $U F$ allied with pattern vector $\alpha$ of the absorbed product. The essential hypothesis in the utility theory is that the choice permanently selects the margins with the maximum utility value. The particulars of the mathematical positions of such function are styled in the resulting.

In the web choice, when assessing the utility of an accessible web, we separate the upward and downward principles related to a net. The measures of which the upper favorite relation is in the service of the upper value are titled upward measures, like to distribute bandwidth, output, consistency degree. Clearly, customers select the upper values of upward principles and inferior values of 
downward principles.Next, we explore the utility formula (based on FNTE) for the upward principles and then cover the outcomes to the downward processes. To define any UF, we must take into account the following measures:

- UF has an upper bound value. This implies that, we can normalize UF to scale the interval [0,1], i.e., $\mu\left(\alpha_{t}\right) \in[0,1]$ (single-pattern utility function);

- UF has first and second derivatives on interval $\left[\alpha_{\min }, \alpha_{\max }\right]$. This returns the information that the utility should not adjustment radically assumed a very small change to a measurable value (product's criteria) and the marginal utility should be fixed;

- UF is a non-decreasing function of $\alpha_{t}$, i.e., the more incomes are owed to the customer. Though, the development of UF is disappearing when the owed incomes reach a certain threshold and greater level of customer fulfillment is found. Mathematically, it must achieve the limit $\lim _{\alpha_{t} \rightarrow \alpha_{\max }} \mu^{\prime}\left(\alpha_{t}\right)=0$. The result of increasing marginal utility infers the concavity of UF;

- The UF necessities can be shortened in the following settings inequalities:

$\mu^{\prime}\left(\alpha_{l}\right) \geq 0, \quad \mu^{\prime \prime}\left(\alpha_{l}\right)>0,($ or $<0)$.

- Also, UF has functional properties which are unique under a monotonic transformation [23]. It gives that employing a monotonic transformation to a UF basically makes additional UF on behalf of the same fondness relation;

- In addition, from the above general necessities, we should remodel the sigmoidal UF to satisfy the following conditions:

$$
\begin{aligned}
& \mu(\alpha)=0, \quad \alpha \leq \alpha_{\min } \\
& \mu(\alpha)=1, \quad \alpha \geq \alpha_{\text {max }} \\
& 0<\mu(\alpha)<1, \quad \alpha \in\left(\alpha_{\text {min }}, \alpha_{\text {max }}\right)
\end{aligned}
$$

\subsection{Single-vision UF}

The central object of this subsection is to define a new UF (we call it Fuzzy Utility Function FUF)and study its properties such as: marginal limit, second derivative, increasing function, concavity and convexity conditions. Our definition is based on the conclusion given in Sect. 2. Let We illustrate the following definition

$\mu(\alpha)=\left(\frac{\bar{\alpha}-\bar{\alpha}_{\min }}{\bar{\alpha}_{\max }-\bar{\alpha}_{\min }}\right)^{\wp}$,

where $\varnothing=p-q>0$. The properties of (2) are given in the next result.
Proposition 3.1 Existence of FUF

Let $0<\bar{\alpha}_{\min } \leq \bar{\alpha}_{1} \leq \bar{\alpha}_{\max }<\infty$ be a variation range of an upward vision $\alpha$. If $\varnothing \rightarrow 0$, then (2) is suitable FUF for $\bar{\alpha}$.

Proof 1 A direct computation, we find that the suggested FUF fulfills the conditions in (1). It remains to show that the marginal and the second derivative are achieving the above conditions.

$$
\begin{aligned}
\lim _{\bar{\alpha} \rightarrow \bar{\alpha}_{\max }} \mu^{\prime}(\alpha) & =\lim _{\bar{\alpha} \rightarrow \bar{\alpha}_{\max }}\left(\left(\frac{\bar{\alpha}-\bar{\alpha}_{\text {min }}}{\bar{\alpha}_{\text {max }}-\bar{\alpha}_{\text {min }}}\right)^{\wp}\right)^{\prime} \\
& =\varnothing \lim _{\bar{\alpha} \rightarrow \bar{\alpha}_{\max }}\left(\frac{\bar{\alpha}-\bar{\alpha}_{\text {min }}}{\bar{\alpha}_{\text {max }}-\bar{\alpha}_{\text {min }}}\right)^{\wp-1} \\
& =\varnothing .
\end{aligned}
$$

On other hand, we have

$$
\begin{aligned}
\lim _{\bar{\alpha} \rightarrow \bar{\alpha}_{\min }} \mu^{\prime}(\alpha) & =\lim _{\bar{\alpha} \rightarrow \bar{\alpha}_{\min }}\left(\left(\frac{\bar{\alpha}-\bar{\alpha}_{\text {min }}}{\bar{\alpha}_{\text {max }}-\bar{\alpha}_{\min }}\right)^{\varnothing}\right)^{\prime} \\
& =\varnothing \lim _{\bar{\alpha} \rightarrow \bar{\alpha}_{\min }}\left(\frac{\bar{\alpha}-\bar{\alpha}_{\min }}{\bar{\alpha}_{\max }-\bar{\alpha}_{\min }}\right)^{\varnothing-1} \\
& =0 .
\end{aligned}
$$

Combining the last two assertions, and taking in account $\wp \rightarrow 0$, we conclude that

$\lim _{\bar{\alpha} \rightarrow \bar{\alpha}_{\max }} \mu^{\prime}(\alpha)=\lim _{\bar{\alpha} \rightarrow \bar{\alpha}_{\min }} \mu^{\prime}(\alpha)=0$.

In addition, the extreme values of $\mu$ are given below

$\mu(\alpha)= \begin{cases}0 & \text { if } \bar{\alpha} \rightarrow \bar{\alpha}_{\text {min }} \\ \left(\frac{\bar{\alpha}-\bar{\alpha}_{\text {min }}}{\bar{\alpha}_{\text {max }}-\bar{\alpha}_{\text {min }}}\right)^{\wp} \in(0,1) & \text { if } 0 \leq \bar{\alpha} \leq 1 \\ 1 & \text { if } \bar{\alpha} \rightarrow \bar{\alpha}_{\text {max }}\end{cases}$

Thus, $\mu$ is continuously differentiable and consequently has a non vanish second derivative. $\mu$ achieves all requests of UF.

Note that the formula of FUF for a downward process is $(1-\mu)$ where $\mu$ upward process. Figure 1 shows the behavior of the proposed FUF. It shows that the best value, when $\wp \in(0,1)$ which satisfies the Sigmoidal function graph.

\subsection{Multi-vision UF}

A multi-pattern utility function (MUF) in the web choice should consider the connections among the reflected 

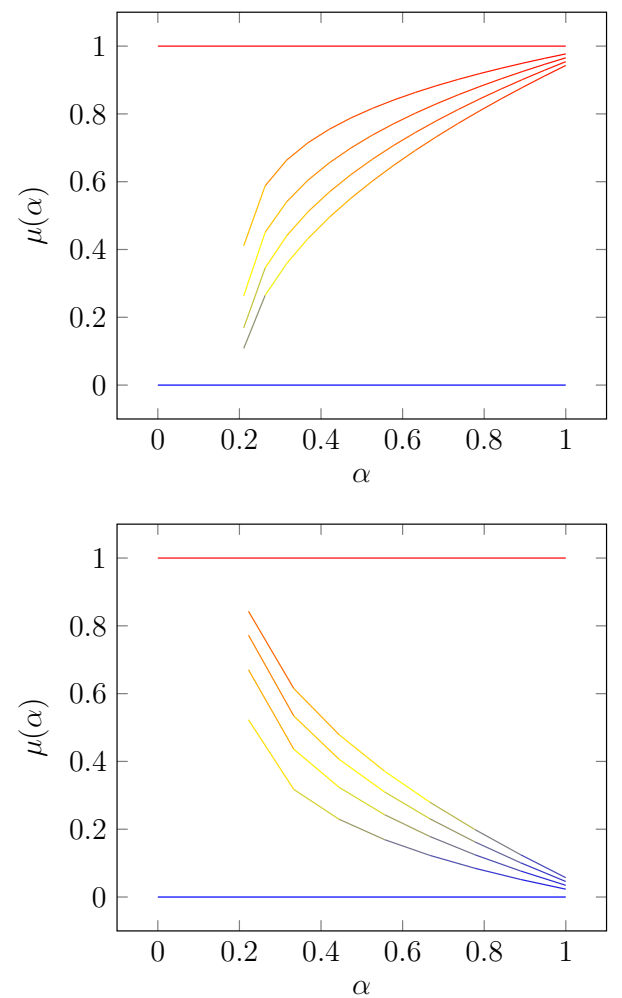

Fig. 1 Single-vision FUF, $\mu(\alpha)$ with different values of $\wp=.2, .3, .4, .5$. upward process and downward process respectively

pattern. An elementary request is the existence such that can be fully recovered by another pattern or from a set of other patterns. In other words, the duffer of a definite fundamental utility does not bring to an exclusion of this access web in the choice process. Usually, when the customer gangs a non-zero favorite weight for a standard, he reflects this standard in its assessment. If its utility is zero, the linked access web does not fulfill the practical and/or customer limitations. Sensibly, this access web should not be nominated. That is rough, the non-zero favorite standards are not independent of each others. Recall that the web's choice in heterogeneous webs is based on multiple standards. A common method to compute the collection MUF of an accessible web is considered in the following approach:

$\mathbb{U}(\alpha)=\sum_{j=1}^{n} \omega_{J} \mu_{J}\left(\alpha_{J}\right)$

where $\alpha$ is a vector of $\mathrm{n}$-pattern (categories, measure or criteria), $\alpha_{j}$ indicates the price of pattern in access $J_{1} \omega_{j}$ represents to the weight of pattern $J$ satisfying $\sum \omega_{J}=1$ and $\mu_{J}$ is normalized function. This formula is normally represented to as an additive utility.
Along with the recognized limits of existing MUF, we want to scheme an original MUF form that fulfills following necessities:

(i) $\frac{\partial \cup(\alpha)}{\partial \mu_{J}} \geq 0, \quad \forall \mu_{j} ;$

(ii) $\operatorname{sign}\left(\frac{\partial \mathbb{U}(\alpha)}{\partial \alpha_{j}}\right)=\operatorname{sign}\left(\mu_{j}^{\prime}\left(\alpha_{\jmath}\right)\right)$;

(iii) $\lim _{\mu_{\jmath} \rightarrow 0} \mathbb{U}(\alpha)=0, \quad \forall \imath=1, \ldots, n$;

(iv) $\lim _{\mu_{l} \rightarrow 1} \mathbb{U}(\alpha)=1, \quad \forall \iota=1, \ldots, n$.

The MUF should increase for increasing single FUF (i), for the aggregate upward principle and reducing downward principle (ii). The conditions (iii) and (iv) return the fact that if all single utilities are seamlessly fulfilled, the MUF should be too. Also, the MUF is an essential formal to get the capacity taking into account the consumer favorite weights for altered standards.

In our discussion, we shall consider the FUF as a single elementary utility for $\alpha_{\text {J }}$. And we aim to achieve all the conditions in (3). Therefore, we design the MUF as follows

$\mathbb{U}(\alpha)=\sum_{l=1}^{n} \omega_{J} \mu_{J}\left(\alpha_{J}\right)$

where $\omega_{J}=\Gamma(1+\wp) \varpi_{J}$ and $\mu$ is given in (2). We have the following existence result:

\section{Proposition 3.2 Existence of MUF}

Consider a web choice principles vector $\alpha$, the related favorite vector $\omega$ and the MUF which is given in (4). If $\mu$ is given in Proposition 3.1 then (4) achieves all the conditions in (3).

Proof 2 Suppose that $\mu_{J}$ takes the formula (2). Then we have

$$
\begin{aligned}
\frac{\partial \cup(\alpha)}{\partial \mu_{J}} & =\frac{\partial\left(\sum_{J=1}^{n} \omega_{J} \mu_{J}\right)}{\partial \mu_{J}} \\
& =\frac{\sum_{J=1}^{n} \partial\left(\omega_{J} \mu_{J}\right)}{\partial \mu_{J}}=\sum_{J=1}^{n} \omega_{J}=1>0 .
\end{aligned}
$$

This showed (i), to prove that $\mathbb{U}(\alpha)$ achieves (ii), a computation brings

$\operatorname{sign}\left(\frac{\partial \cup(\alpha)}{\partial \mu_{\imath}}\right)=\lim _{n \rightarrow \infty} \sum_{j=1}^{n} \omega_{J}=\sum_{j=1}^{n} \Gamma(1+\varnothing) \lim _{n \rightarrow \infty} \varpi=0$. 
Fig. 2 The variation of MUF using $\varnothing=0.2$ (left) and $\wp=0.5$ (right). The function is convex and for $(1-\mathbb{U}(\alpha))$ is concave
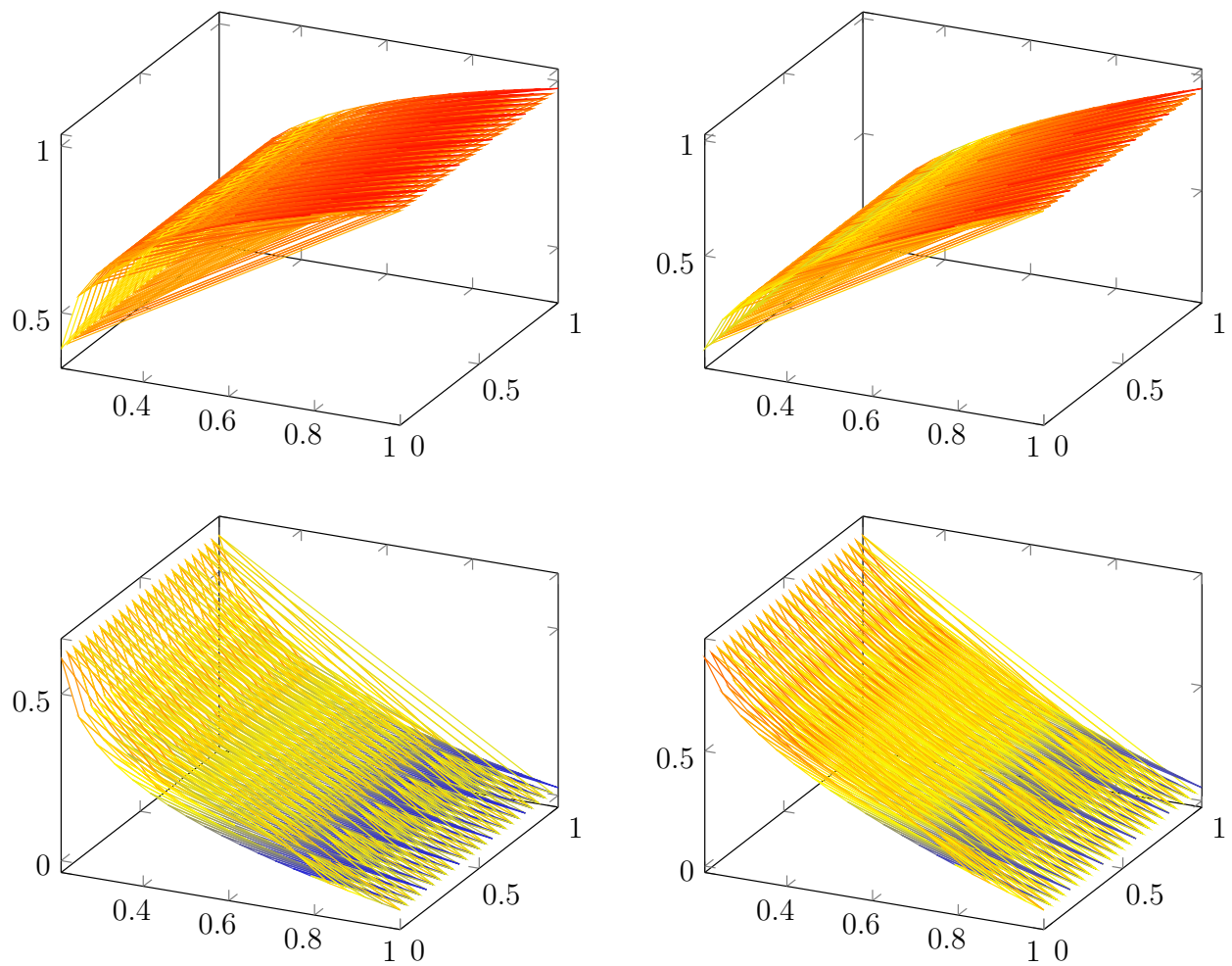

On the other hand, by Proposition 3.1, we have showed that $\lim _{\mu(\alpha) \rightarrow \wp_{\max }} \mu_{j}^{\prime}\left(\alpha_{j}\right)=0$ for all $\alpha_{j}$; thus we conclude that

$\operatorname{sign}\left(\frac{\partial \mathbb{U}(\alpha)}{\partial \mu_{\jmath}}\right)=\operatorname{sign}\left(\mu_{J}^{\prime}\left(\alpha_{\jmath}\right)\right)=0$

$$
\begin{aligned}
\frac{\partial \mathbb{U}(\alpha)}{\partial \alpha_{J}} & =\frac{\partial\left(\sum_{J=1}^{n} \omega_{J} \mu_{J}\left(\alpha_{J}\right)\right)}{\partial \alpha_{J}} \\
& =\frac{\sum_{J=1}^{n}\left(\omega_{J} \partial \mu_{J}\right)}{\partial \alpha_{J}}=\sum_{J=1}^{n} \omega_{J} \mu^{\prime}\left(\alpha_{J}\right),
\end{aligned}
$$

Moreover, by the definition (4), we obtain

which leads to $\frac{\partial \mathbb{U}(\alpha)}{\partial \alpha_{j}} \geq 0$ for $0 \leq \wp<1$. This shows that

$$
\begin{aligned}
\lim _{\mu_{J} \rightarrow 0} \mathbb{U}(\alpha) & =\lim _{\mu_{J} \rightarrow 0} \sum_{J=1}^{n} \omega_{J} \mu_{J}\left(\alpha_{J}\right) \\
& =\sum_{J=1}^{n} \lim _{\mu_{J} \rightarrow 0}\left(\omega_{J} \mu_{J}\left(\alpha_{J}\right)\right)=0, \quad \forall J=1, \ldots, n .
\end{aligned}
$$

$\operatorname{sign}\left(\frac{\partial \mathbb{U}(\alpha)}{\partial \alpha_{J}}\right)=\operatorname{sign}\left(\mu_{J}^{\prime}\left(\alpha_{J}\right)\right)$

that is is MUF is increasing for upward principle and decreasing for downward principle. The proof is completed.

A calculation implies that

$$
\begin{aligned}
\lim _{\phi_{l} \rightarrow 1} \mathbb{U}(\alpha) & =\lim _{\phi_{l} \rightarrow 1} \sum_{l=1}^{n} \omega_{l} \phi_{l}\left(x_{l}\right) \\
& =\sum_{l=1}^{n} \lim _{\phi_{l} \rightarrow 1}\left(\omega_{l} \phi_{l}\left(x_{l}\right)\right)=\sum_{l=1}^{n} \omega_{l}=1 .
\end{aligned}
$$

Figure 2 shows the variation of the acceptance probability. Our original suggested MUF can be returned accurately the approval probability for the radio supply management. Note that the offered MUF formula is not certainly the unique one, since its monotonic transformation creates extra MUF and its definition comes from fuzzy function (FUF) but it is the one that fulfills all our requests.

Finally, we have 


\subsection{Numerical examples}

Suppose that the network selection is found on only two principles: distributed bandwidth $\beta$ and paid cost $\alpha$. The single-vision utility is recognized based on the parameters assumed in Table 2 . The assumed values of cost and bandwidth are the qualified ones thus they are not needed to have a unit. Note also that we utilize the form (2). In view of Example 2.1, the fractal operator $\Delta^{0.5}\left(\alpha_{1}, \alpha_{2}, \alpha_{3}\right) \approx 1$ that is the construction of data in Example 2.1 achieves the maximization of the selecting. If we let $\alpha_{i}=0.1$ under the same $\beta_{i}$ and $\omega_{i}$ in Example 2.1 for all networks, then we obtain the following distributions $\Delta^{0.1}\left(\alpha_{1}, \alpha_{2}, \alpha_{3}\right)=0.1^{0} .1 * 0.5+0.1^{0} .1 * 0.2+0.1^{0} .1 * 0.3=0.7943$, $\Delta^{0.5}\left(\alpha_{1}, \alpha_{2}, \alpha_{3}\right)=0.1^{0} .5 * 0.5+0.1^{0} .5 * 0.2+0.1^{0} .5 * 0.3=0.3162$, and $\Delta^{0.8}\left(\alpha_{1}, \alpha_{2}, \alpha_{3}\right)=0.1^{0} .8 * 0.5+0.1^{0} .8 * 0.2+0.1^{0} .8 * 0.3=0.1584$, which means that the selection on this web is not valid for the customer. Other distribution is given as follows: $\Delta^{0.1}(0.1,0.2,0.9)=0.1^{0} .1 * 0.5+0.2^{0} .1 * 0.2+0.9^{0} .1 * 0.3=0.864$, $\Delta^{0.5}(0.1,0.2,0.9)=0.1^{0} .5 * 0.5+0.2^{0} .5 * 0.2+0.9^{0} .5 * 0.3=0.5321$, and $\Delta^{0.8}(0.1,0.2,0.9)=0.1^{0} .8 * 0.5+0.2^{0} .8 * 0.2+0.9^{0} .8 * 0.3=0.4101$. Also, cannot reach the maximization of the selection. If the parameters satisfy the following distribution $\alpha_{i}=0.5, w p=0.5$, then we obtain $\Delta^{0.5}(0.5,0.5,0.5)=0.5^{0} .5 * 0.5+0.5^{0} .5 * 0.2+0.5^{0} .5 * 0.3=0.707$, this leads that the best selection fits Example 2.1. The last distribution of equal cost values does not achieve the best selection compering with the $\Delta^{0.5}(0.5,0.6,0.7)=1$. Moreover, if we consider high cost $\alpha_{i}=0.99$, we obtain $\Delta^{0.1}(0.99,0.99,0.99) \simeq \Delta^{0.5}(0.99,0.99,0.99) \simeq \Delta^{0.8}(0.99,0.99,0.99) \approx 1$, with optimal utility of the web and best selection, but the cost is high compering with Example 2.1.

\section{Discussion}

The real time video and voice use with continual bit rate are rigid in their request for bandwidth. Their utility is displayed as a step function with only two prices, either fulfilled or unfulfilled, depending on whether the assigned bandwidth is above or below a specified threshold. Therefore, the suggested fuzzy utility function is a good selection. This type of UF is originally linked with the aggregate of bandwidth that the web potentials to deputise for a usage gathering in the online Internet location. We approve that inelastic requests are very utilitarian to the variation of the supply division and the utility forming should take into account such indifference. Nonetheless, the usage of the step utility functions in the wireless setting, and mainly in the web choice, is not suitable. For further investigation in the future, we have the expectation to develop more generalizations of these operators by utilizing other characteristics, in the decision process, such as uncertain information, fractional entropy and more complex structures. Other decision making problems and other applications will also be assumed.

\section{Conclusion}

FGO its alternative generalizations (including fuzzy type) are given a high score in all applications in different sciences. Very newly, a new feature of the FGO, called fuzzy FGO is delivered in the utility theory. The effort has designed a model of the single-pattern utility function as well as the multi-vision utility in the setting of the wireless access web choice. This adaptation takes into account the performances of mobile communicating end-consumers. The boundaries of present existing utility simulations have been spotted. The FUF and MUF (sigmoid function) operating forms, which have the skills to fulfill all the utility properties and to improve the revealed problems of obtainable models, have been suggested. The fittings and success of the suggested design have been certified through statistical analysis and mathematical modeling.

Acknowledgements The author wishes to express her profound gratitude to the anonymous referees and the editors for their careful reading of the manuscript and the very useful comments that have been implemented in the final version of the manuscript.

\section{Compliance with ethical standards}

Conflict of interest The author declares that there is no conflict of interest.

\section{References}

1. Nguyen $V$ et al (2008) On utility models for access network selection in wireless heterogeneous networks. In: NOMS 2008 IEEE network operations and management symposium. IEEE

2. Von Neumannn J, Morgenstern O (1953) Theory of games and economics behavior. Princeton University Press, Princeton, NJ

3. Salih YK et al (2015) A user-centric game selection model based on user preferences for the selection of the best heterogeneous wireless network. Ann Telecommun 70(5-6):239-248

4. Salih YK et al (2016) An intelligent selection method based on game theory in heterogeneous wireless networks. Trans Emerg Telecommun Technol 27(12):1641-1652

5. Wang HJ, Katz RH, Giese J (1999) Policy-enabled handoffs across heterogeneous wireless networks. In: IEEE workshop on mobile computing systems and applications' 99 , Louisiana

6. Chen L, Sun T, Chen B, Rajendran V, Gerla M (2004) A smart decision model for vertical handoff. In: Proceedings of 4 th ANWIRE, Greece

7. Ormond O, Muntean G, Murphy J (2005) Network selection decision in wireless heterogeneous networks. In: Proceedings of IEEE 16th international symposium on personal, indoor and mobile radio communications, Berlin 
8. Ibrahim RW, Jalab H, Gani A (2015) Cloud entropy management system involving a fractional power. Entropy 18(1):14

9. Ibrahim RW, Ghani A (2016) Hybrid cloud entropy systems based on Wiener process. Kybernetes 45:1072-1083

10. Ibrahim RW, Jalab H, Gani A (2016) Perturbation of fractional multi-agent systems in cloud entropy computing. Entropy 18(1):31

11. Ormond O, Muntean G, Murphy J (2006) Utility-based intelligent network selection in beyond $3 G$ systems. In: Proceedings of ICC, Turkey

12. Vecer J (2019) Dynamic scoring: probabilistic model selection based on utility maximization. Entropy 21(1):36

13. Badia L, Zorzi M (2004) On utility-based radio resource management with and without service guarantees. In: Proceedings of the 7th ACM international symposium on modeling, analysis and simulation of wireless and mobile systems. ACM Press, New York, pp 244-251

14. He YD, He Z (2016) Extensions of Atanassov's intuitionistic fuzzy interaction Bonferroni means and their application to multipleattribute decision making. IEEE Trans Fuzzy Syst 24:558-573

15. Marichal JL (1998) Aggregation operators for multicriteria decision aid. Unpublished Ph.D. dissertation, Dept Manag, Inst Math Univ Liege, Liege, Belgium

16. Aristondo $O$ et al (2012) The Gini index, the dual decomposition of aggregation functions, and the consistent measurement of inequality. Int J Intell Syst 27(2):132-152
17. Yampolskiy RV (2014) Utility function security in artificially intelligent agents. J Exp Theor Artif Intell 26(3):373-389

18. Weihua Ou et al (2017) Patch-based visual tracking with online representative sample selection. J Electron Imaging 26(3):033006. https://doi.org/10.1117/1.JEl.26.3.033006

19. Yuan $D$ et al (2019) Particle filter re-detection for visual tracking via correlation filters. Multimed Tools Appl 78(11):14277-14301

20. Yuan D et al (2019) A multiple feature fused model for visual object tracking via correlation filters. Multimed Tools Appl $1: 1-20$

21. Chiclana F, Herrera F, Herrera-Viedma E (2000) The ordered weighted geometric operator: properties and applications. In: Proceeding 8th international conference on information processing and management of uncertainty in knowledge-based systems. Madrid Spain, vol 22, pp 985-991

22. Yang XJ, Baleanu D, Srivastava HM (2015) Local fractional integral transforms and their applications. Academic Press, Cambridge

23. Essays UK (2018) Monotonic transformation of utility function. Retrieved fromhttps://www.ukessays.com/essays/economics/ monotonic-transformation-utility-3581.php?vref=1

Publisher's Note Springer Nature remains neutral with regard to jurisdictional claims in published maps and institutional affiliations. 